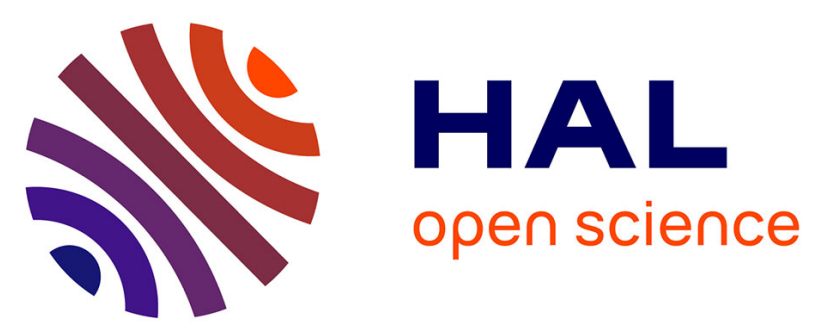

\title{
Les usages du brevet: vers une définition du brevet comme mécanisme de gouvernance des activités d'innovation des organisations
}

\author{
Matthieu Mandard
}

\section{- To cite this version:}

Matthieu Mandard. Les usages du brevet: vers une définition du brevet comme mécanisme de gouvernance des activités d'innovation des organisations. Innovations - Revue d'économie et de management de l'innovation, 2018, 3 (57), pp.165-187. 10.3917/inno.pr1.0048 . halshs-02344777

\section{HAL Id: halshs-02344777 \\ https://shs.hal.science/halshs-02344777}

Submitted on 16 Apr 2021

HAL is a multi-disciplinary open access archive for the deposit and dissemination of scientific research documents, whether they are published or not. The documents may come from teaching and research institutions in France or abroad, or from public or private research centers.
L'archive ouverte pluridisciplinaire HAL, est destinée au dépôt et à la diffusion de documents scientifiques de niveau recherche, publiés ou non, émanant des établissements d'enseignement et de recherche français ou étrangers, des laboratoires publics ou privés. 


\title{
Les usages du brevet : vers une définition du brevet comme mécanisme de gouvernance des activités d'innovation des organisations
}

\author{
Matthieu MANDARD \\ Maître de conférences en sciences de gestion \\ Université Rennes 1 \\ Centre de Recherche en Économie et Management (CREM - UMR CNRS 6211) \\ 11 rue Jean Macé - CS 70803 - 35708 RENNES Cedex 7 - FRANCE \\ E-mail : matthieu.mandard@univ-rennes1.fr
}

Résumé : L'objectif de cet article est de proposer un cadre analytique qui répertorie les usages du brevet. Pour ce faire, nous proposons de remettre en question la définition traditionnelle du brevet comme mécanisme de protection des innovations pour le définir plus largement comme un mécanisme de gouvernance des activités d'innovation des organisations, et ce au niveau intra- comme inter-organisationnel. Nous montrons ainsi que le brevet a quatre principaux usages - indicateur d'activité, outil de motivation, vecteur de réputation et mécanisme de protection - dont nous présentons les modalités de mise en œuvre ainsi que les limites. Nous discutons finalement les implications scientifiques et managériales de ce cadre d'analyse.

Mots clés : Brevet ; gouvernance ; innovation; motivation ; propriété intellectuelle ; réputation. 
Les usages du brevet ont fait l'objet d'une attention considérable au cours des dernières décennies. Par-delà son rôle originel de mécanisme de protection des innovations, le brevet s'est en effet révélé avoir de nombreuses fonctionnalités, telles que transmettre la réputation des inventeurs, générer des revenus au travers de cessions de licences, surveiller les compétiteurs, manager les activités de R\&D en interne, etc. De nos jours, il est ainsi considéré comme une ressource particulièrement polyvalente.

Alors que de très nombreuses revues de la littérature existent sur ce sujet (voir par exemple Blind, Edler, Frietsch, \& Schmoch, 2006; Corbel, Fernandez, \& Gendraud, 2007; Corbel \& Le Bas, 2011; Ernst, 2003; Graham \& Sichelman, 2008; Holgersson, 2013; Somaya, 2012), on ne dispose toujours pas d'un cadre analytique qui répertorie ces usages de manière exhaustive. Si des distinctions entre usages internes et externes (Corbel et al., 2007; Ernst, 2003), ou entre usages stratégiques et autres usages (Somaya, 2012) ont bien été proposées, une classification systématique n'a en effet pas encore été établie.

Ceci pose deux problèmes. D'un point de vue scientifique tout d'abord, l'absence d'un tel cadre analytique empêche de délimiter les usages du brevet en tant que champ de recherche, et les zones qui nécessitent de nouvelles investigations ne peuvent être identifiées de manière exhaustive. D'un point de vue managérial ensuite, cette absence freine la popularisation de ces différents usages, alors même que des voix se sont élevées pour dénoncer la fréquente méconnaissance des détenteurs de brevets en la matière - en particulier en ce qui concerne les petites et moyennes entreprises (Blind et al., 2006; Pitkethly, 2012).

L'objectif de l'article est donc de s'attaquer à cette problématique en proposant un cadre analytique qui répertorie les usages du brevet de manière exhaustive. Pour ce faire, nous proposons de remettre en question la définition traditionnelle du brevet comme mécanisme de protection des innovations pour le définir plus largement comme un mécanisme de gouvernance des activités d'innovation des organisations, et ce au niveau intra- comme interorganisationnel. Nous montrons ainsi que le brevet a quatre principaux usages - indicateur d'activité, outil de motivation, vecteur de réputation et mécanisme de protection - dont nous présentons les modalités de mise en œuvre ainsi que les limites ${ }^{1}$. Nous discutons finalement les implications scientifiques et managériales de ce cadre d'analyse.

\section{UNE PROPOSITION DE REMISE EN QUESTION DE LA DÉFINITION TRADITIONNELLE DU BREVET}

La création du brevet au 15ème siècle fut motivée par la volonté de protéger les innovations contre l'imitation (P. O. Long, 1991). L'objectif était de promouvoir l'innovation en fournissant aux inventeurs un intervalle de temps au cours duquel ils pourraient bénéficier d'un retour sur leurs investissements. Depuis lors, cependant, un nouveau contexte économique et managérial est apparu - de grandes organisations ont émergé, l'intensité concurrentielle s'est accrue, l'innovation ouverte est devenue fréquente (Chesbrough, 2003) et les usages du brevet ont évolué. Pourtant, en raison de sa vocation historique et de sa désignation en tant que mécanisme de propriété intellectuelle, le brevet est encore souvent considéré à l'aune de son rôle de protection.

Nous suggérons ici que, en accord avec l'évolution du contexte économique et managérial, l'usage du brevet a basculé d'un simple rôle de mécanisme de protection vers un rôle plus

1 Puisque l'objectif de l'article est de proposer une classification des usages du brevet, il convient de noter qu'il n'entre pas de son propos de réaliser une revue de la littérature de leurs antécédents et de leurs conséquences respectives (sur ce point, voir par exemple Corbel \& Le Bas, 2011; Somaya, 2012). 
général de mécanisme de gouvernance des activités d'innovation des organisations, et ce au niveau intra- comme inter-organisationnel.

Qu'est-ce qu'un dispositif de gouvernance? Nous le définirons comme un arrangement organisationnel qui permet d'assurer la coordination et le contrôle des activités d'un collectif, deux dimensions qui sont souvent considérées comme les problèmes majeurs auxquels les organisations doivent faire face (Marsden, Cook, \& Kalleberg, 1994) ${ }^{2}$.

$\mathrm{Au}$ sens où nous l'entendons ${ }^{3}$, la coordination relève de l'agencement des activités des membres d'un collectif. Il s'agit de structurer les activités de chacun en direction des objectifs fixés, en établissant des plans d'action et en répartissant les tâches respectives. Le contrôle relève quant à lui de l'incitation des membres d'un collectif à réaliser les activités qui leur sont attribuées. Il s'agit ici de s'assurer que les efforts de chacun sont effectivement déployées en direction les objectifs qui lui sont fixés, en attribuant des récompenses ou en prévoyant des pénalités.

Afin de surmonter ces problèmes de coordination et de contrôle, des mécanismes de gouvernance tels que des contrats de travail ou des règlements qui spécifient les tâches et les incitations sont ainsi mis en œuvre au sein des entreprises. Il est intéressant de noter que les brevets peuvent eux aussi être vus comme des mécanismes de gouvernance, dans la mesure où ils sont de nos jours employés par les organisations pour tout à la fois coordonner et contrôler leurs activités d'innovation ${ }^{4}$.

Au niveau intra-organisationnel, le brevet a en effet deux principaux usages : coordonner les activités d'innovation des organisations en tant qu'indicateur d'activité, et contrôler les activités d'innovation des organisations en tant qu'outil de motivation. En matière de coordination, le brevet permet d'identifier les inventeurs et d'établir des programmes de R\&D en conséquence. En matière de contrôle, le brevet est par ailleurs souvent associé à divers dispositifs incitatifs et permet ainsi d'encourager les employés à innover.

Au niveau inter-organisationnel, le brevet a également deux principaux usages : coordonner les activités d'innovation des organisations en tant que vecteur de réputation, et contrôler les activités d'innovation des organisations en tant que mécanisme de protection - ce qui correspond à sa vocation historique. En matière de coordination, le brevet permet en effet d'identifier les entreprises innovantes au sein d'un secteur d'activité, ce qui permet aux organisations d'ajuster mutuellement leurs activités d'innovation respectives. En matière de contrôle, puisque le brevet confère des droits de propriété intellectuelle, il permet aux organisations qui en sont détentrices d'autoriser ou d'empêcher la mise en œuvre d'activités d'innovation par des organisations concurrentes.

Ces quatre principaux usages sont recensés dans le Tableau 1.

2 Le concept de gouvernance fait l'objet de multiples acceptions en sciences de gestion. La définition que nous proposons ici a été retenue car elle appuyée par de nombreux travaux (Edström \& Galbraith, 1977; Gupta, Dirsmith, \& Fogarty, 1994; Kumar \& Seth, 1998; Malhotra \& Lumineau, 2011), et parce qu'elle s’avère particulièrement opérante pour bien mettre en évidence les principaux usages du brevet.

3 Il nous faut également souligner que les concepts de coordination et de contrôle font eux aussi l'objet de multiples définitions, c'est pourquoi il convient de se rapporter à celles que nous employons ici et qui reprend celles de Marsden et alii (1994).

4 L’usage du brevet comme mécanisme de coordination a d’ailleurs été largement souligné par le passé (voir notamment Cohendet, Farcot, \& Pénin, 2006; Pénin, 2005). 
Tableau 1 - Les principaux usages du brevet

Au niveau intra-

Au niveau interorganisationnel organisationnel

\begin{tabular}{lcc}
$\begin{array}{l}\text { En tant que mécanisme de } \\
\text { coordination }\end{array}$ & $\begin{array}{c}\text { Un indicateur } \\
\text { d'activité }\end{array}$ & $\begin{array}{c}\text { Un vecteur de } \\
\text { réputation }\end{array}$ \\
\hline $\begin{array}{l}\text { En tant que mécanisme de } \\
\text { contrôle }\end{array}$ & $\begin{array}{c}\text { Un outil de } \\
\text { motivation }\end{array}$ & $\begin{array}{c}\text { Un mécanisme de } \\
\text { protection }\end{array}$
\end{tabular}

\section{LES USAGES DU BREVET AU NIVEAU INTRA-ORGANISATIONNEL}

Ainsi que mentionné plus haut, au niveau intra-organisationnel, le brevet peut être employé afin de gouverner les activités d'innovation des organisations en interne. Dans cette perspective, le brevet a deux usages principaux qui sont régulièrement occultés : coordonner les activités d'innovation des organisations en tant qu'indicateur d'activité, et contrôler leurs activités d'innovation en tant qu'outil de motivation.

\section{Le brevet comme indicateur d'activité}

En tant qu'il permet d'identifier les inventeurs et les inventions au sein d'une organisation, le brevet peut tout d'abord être employé comme indicateur d'activité. Il permet ici de coordonner les efforts de l'organisation en matière d'innovation en établissant des programmes de $R \& D$ et en précisant les rôles de chacun. C'est à ce titre que le brevet est souvent présenté comme un important outil de management des connaissances (Ernst, 2003).

\section{Benchmarking interne}

Les brevets sont susceptibles de favoriser la coordination des activités de R\&D dans la mesure où ils autorisent l'identification des sources d'innovation au sein d'une organisation. Puisqu'ils sont délivrés par des offices de brevet qui suivent des procédures standardisées, ils constituent en effet un indicateur apprécié.

Premièrement, au niveau individuel, les brevets permettent l'identification des employés les plus inventifs ainsi que l'identification des détenteurs de connaissances particulières (Ernst, 2003; Moehrle, Walter, Geritz, \& Müller, 2005). Ceci peut être d'une importance particulière lorsque les développements technologiques d'une organisation sont fortement concentrés autour de quelques individus, ce qui en rend les organisations fortement dépendantes (Ernst, Leptien, \& Vitt, 2000). L'identification de ces employés innovants peut ainsi favoriser la coordination des activités de $\mathrm{R} \& \mathrm{D}$ au sein des organisations, dans la mesure où elle peut servir à l'organisation du travail - afin de positionner au mieux les employés innovants en fonction de leurs compétences -, où à la mise en place de plans de formations - afin de maintenir ou de développer les compétences des employés innovants (Moehrle et al., 2005). 
Deuxièmement, au niveau de l'organisation toute entière, les brevets peuvent également servir à identifier les services de R\&D les plus performants afin d'encourager ou, inversement, afin d'abandonner certaines activités (Ernst, 2003). Ceci peut être particulièrement utile dans les grandes organisations qui comprennent de multiples centres R\&D qui investiguent différentes aires technologiques. En France, le brevet est ainsi l'un des indicateurs utilisés par le Centre National de la Recherche Scientifique afin d'évaluer la productivité de ses unités de recherche.

\section{Benchmarking externe}

Outre les comparaisons au sein des organisations, les brevets peuvent également permettre d'organiser les activités de $R \& D$ en interne en autorisant des comparaisons entre les organisations - il convient de noter ici que, bien que basé sur une analyse conduite au niveau inter-organisationnel, cet usage a bel et bien vocation à organiser l'innovation en interne. Ils constituent un indicateur d'activité particulièrement utile dans la mesure où ils fournissent une information précise quant aux pratiques de R\&D des concurrents, laquelle ne serait pas disponible si les inventions avaient été tenues secrètes (Ernst, 1998). De la même manière qu'au niveau intra-organisationnel, deux niveaux d'analyse peuvent être distingués ici.

Premièrement, au niveau individuel, les brevets sont régulièrement employés afin de localiser et de recruter des inventeurs externes (Almeida \& Kogut, 1999; Moehrle et al., 2005). En conséquence, ceci conduit fréquemment les organisations susceptibles d'être victimes de ces débauchages à développer des politiques de restriction de la mobilité professionnelle afin de limiter les départs (Delerue \& Lejeune, 2010), ou à développer une réputation de pugnacité en matière de défense de leurs droits de propriété intellectuelle afin de prévenir les fuites de connaissances de la part des employés recrutés par la concurrence (Agarwal, Ganco, \& Ziedonis, 2009; Ganco, Ziedonis, \& Agarwal, 2015).

Deuxièmement, à l'échelle d'une organisation, les portefeuilles de brevets peuvent également permettre aux organisations d'évaluer le paysage technologique d'un secteur: les technologies existantes, leurs détenteurs, leurs origines, leurs évolutions, leur profitabilité (Fabry, Ernst, Langholz, \& Köster, 2006; Lee, Yoon, Lee, \& Park, 2009; Lee, Yoon, \& Park, 2009). Les brevets peuvent ainsi être utilisés afin de comparer la performance technologique d'une organisation par rapport à celle de ses compétiteurs, ce qui peut permettre de lancer, d'accentuer, ou même d'abandonner des activités relatives à un secteur technologique particulier ${ }^{5}$. Depuis la fin des années 1990, les fusions et acquisitions ont été de plus en plus utilisées afin d'acquérir des compétences technologiques externes (De Man \& Duysters, 2005), et le brevet a constitué un outil d'évaluation précieux pour juger de l'opportunité de ces manœuvres stratégiques (Ernst, 2003).

Si le brevet peut être un indicateur d'activité utile, il convient de noter que cet usage souffre d'un certain nombre de limitations. Premièrement, le brevet ne reflète que partiellement les activités d'innovation, dans la mesure où toutes les inventions ne sont pas brevetées (Nelson, 2009). Deuxièmement, le brevet ne reflète pas toujours la contribution d'un inventeur, dans la mesure où des facteurs sociaux tels que la position hiérarchique sont susceptibles d'affecter son attribution (Haeussler \& Sauermann, 2013). Enfin, troisièmement, le brevet ne garantit pas la rentabilité économique des inventions, dans la mesure où la majorité des brevets est de

5 Sur ce point, il convient de souligner que le concept d’inventing around a été spécifiquement développé pour désigner la pratique qui consiste à proposer une invention alternative à une invention brevetée par un concurrent. 
peu de valeur économique (Ernst \& Omland, 2011; Nelson, 2009) ${ }^{6}$, et parce que les organisations tentent parfois de conduire leurs concurrents vers de fausses pistes en brevetant des inventions dans des secteurs technologiques qu'elles savent non profitables (Langinier, 2005). Pour toutes ces raisons, le recours au brevet comme indicateur d'activité doit être mis en œuvre avec précaution.

\section{Le brevet comme outil de motivation}

$\mathrm{Au}$ niveau intra-organisationnel, outre son usage en tant qu'indicateur d'activité, le brevet peut également être employé comme outil de motivation afin d'orienter les efforts des employés vers l'innovation. Il s'agit donc ici de contrôler la réalisation des activités de R\&D. Deux types de motivation sont communément distinguées : une motivation intrinsèque et une motivation extrinsèque (Ryan \& Deci, 2000). Elles peuvent toutes deux être induites par le recours au brevet.

\section{Motivation intrinsèque}

La motivation intrinsèque est définie comme le fait de réaliser une activité pour l'intérêt inhérent que l'on en retire (Ryan \& Deci, 2000). Ce type de motivation est particulièrement important en matière d'innovation, dans la mesure où il a été montré depuis longtemps qu'il joue un rôle décisif dans la créativité individuelle (Amabile, 1983).

Bien que cela ait été étonnamment peu souligné dans la littérature, chez les inventeurs, le brevet peut jouer un rôle médiateur dans le déclenchement d'une motivation intrinsèque dans la réalisation de leur travail. En tant que marqueur de leur contribution aux activités d'innovation de leur organisation, il permet en effet aux inventeurs d'en apprécier l'importance et de stimuler leur intérêt en la matière. C'est ainsi qu'une étude menée chez le fabricant automobile PSA Peugeot-Citroën a récemment montré que, dans une grande organisation telle que celle-ci qui compte environ 200000 employés, les brevets peuvent permettre aux inventeurs de mieux déterminer leur contribution à la conception d'un nouveau véhicule, accentuant de la sorte leur sentiment d'accomplissement professionnel (Chevreuil, Corbel, \& Mbongui-Kialo, 2012).

\section{Motivation extrinsèque}

La motivation intrinsèque est définie comme le fait de réaliser une activité pour des objectifs qui sont distincts de l'activité en elle-même (Ryan \& Deci, 2000). De manière similaire à la motivation intrinsèque, le brevet peut également jouer un rôle médiateur dans le déclenchement d'une motivation extrinsèque à la réalisation d'un travail, en permettant d'assortir des récompenses à la réalisation d'une activité inventive.

Premièrement, le brevet peut être associé à une récompense monétaire. Ainsi, l'un des principaux déposants de brevets français, l'entreprise PSA Peugeot-Citroën précédemment mentionnée, a mis en place pour ses employés un système de primes associées au dépôt de brevets, lesquelles varient en fonction de l'usage que fera l'entreprise de ces brevets. Une prime est attribuée aux inventeurs lorsque le brevet est déposé en France, une autre lorsque

6 Ainsi que le souligne justement un évaluateur de l'article, un brevet peut couvrir aussi bien une invention majeure qu'une petite amélioration incrémentale, c'est la raison pour laquelle les études utilisant les brevets comme indicateurs d'activité intègrent aussi fréquemment les citations de ces derniers dans d'autres brevets comme mesure de leur qualité. 
l'invention est effectivement exploitée par l'entreprise, une autre enfin est offerte lorsque les brevets sont déposés à l'étranger (Chevreuil et al., 2012). L'attribution d'une rémunération complémentaire aux inventeurs en cas de brevetabilité de leur invention ou de dépôts effectif de brevet est d'ailleurs une obligation légale en vigueur dans un certain nombre de pays - e.g., Allemagne, Chine, Danemark, France ${ }^{7}$ ou Russie.

Deuxièmement, le brevet peut également jouer un rôle en matière de renforcements extrinsèques dans la mesure où il permet de signaler les compétences des inventeurs auprès de leurs collègues, renforçant ainsi leur prestige et leur réputation. Certaines entreprises l'ont d'ailleurs bien compris qui communiquent en interne sur les employés les plus inventifs afin de susciter cette motivation extrinsèque. Des études menées auprès de chercheurs universitaires ont même récemment montré que cette récompense intangible est susceptible de constituer un facteur de motivation plus important que la recherche de gains financiers (Baldini, Grimaldi, \& Sobrero, 2007; Göktepe-Hulten \& Mahagaonkar, 2010).

Bien qu'il puisse représenter un dispositif de contrôle utile, le rôle du brevet comme outil de motivation comporte deux limites, qui sont liées à des problématique de justice organisationnelle. D'une part, il peut y avoir un problème de justice procédurale, c'est-à-dire d'équité perçue dans les modalités d'attribution des rétributions des inventeurs (Greenberg, 1990). Ainsi que nous l'avons mentionné précédemment, l'identification des inventeurs d'un brevet peut en effet être le résultat de facteurs socio-politiques dans la mesure où, par-delà la contribution à un projet de $\mathrm{R} \& \mathrm{D}$, des facteurs tels que la position hiérarchique peuvent également influencer l'attribution d'un brevet (Haeussler \& Sauermann, 2013). En conséquence, les compensations associées aux brevets peuvent être perçues comme étant attribuées de manière inappropriée. D'autres part, il peut également y avoir un problème de justice distributive, c'est-à-dire d'équité perçue dans le montant des rétributions attribuées aux inventeurs (Greenberg, 1990). Lorsque de multiples inventeurs sont associés à un brevet, le partage des rétributions peut considérablement réduire les bénéfices individuels (Harhoff \& Hoisl, 2007). En conséquence, les compensations associées aux brevets peuvent être perçues comme insuffisantes. En raison de ces deux problèmes, les compensations peuvent être vues comme injustes et l'usage du brevet comme outil de motivation peut voir son efficacité amoindrie.

\section{LES USAGES DU BREVET AU NIVEAU INTER-ORGANISATIONNEL}

Outre ses usages au niveau intra-organisationnel, le brevet peut également être employé afin de gouverner les activités d'innovation des organisations au niveau inter-organisationnel. Et, de la même manière qu'au niveau intra-organisationnel, le brevet a également deux fonctions principales à ce niveau : coordonner les activités d'innovation des organisations en tant que vecteur de réputation, et contrôler les activités d'innovation des organisations en tant que mécanisme de protection. Plus spécifiquement, le brevet peut permettre aux organisations d'obtenir un avantage compétitif, d'éviter un désavantage compétitif, ou de développer des échanges inter-organisationnels, à la fois en tant que mécanisme de protection - ce qui a déjà été souligné par Somaya (2012) -, mais également en tant que vecteur de réputation (Tableau 2).

7 Il convient d'apporter une précision sur ce point : en droit français, la nature de la contrepartie financière varie selon que l’invention a été réalisée ou non dans le cadre de la mission du salarié. 
Tableau 2 - Les usages du brevet au niveau inter-organisationnel

Le brevet comme vecteur de réputation
Le brevet comme mécanisme de protection
Obtenir un avantage compétitif
Stratégie de signal
Stratégie propriétaire

Éviter un désavantage compétitif Stratégie de légitimation Stratégie défensive

Stratégie collaborative Stratégie de négociation

\section{Le brevet comme vecteur de réputation}

Dans la mesure où il porte des inventions à la connaissance du public, le brevet transmet aux constituants de l'environnement d'une organisation des informations quant à ses compétences technologiques, servant ainsi de vecteur de réputation. Ce faisant, il permet aux organisations et à leurs interlocuteurs de coordonner leurs efforts en matière d'innovation. Trois stratégies génériques peuvent être identifiées ici.

\section{La stratégie de signal}

La stratégie de signal correspond à l'usage du brevet en tant que vecteur de réputation afin d'influencer positivement les constituants de l'environnement d'une organisation, ceci afin de lui offrir un avantage compétitif. En situation de compétition intensive, le brevet peut en effet permettre aux organisations de se différencier en affichant des compétences technologiques supérieures à celles de leurs concurrents. Il convient de noter ici que la définition de la stratégie de signal proposée est susceptible de différer de celle proposée par d'autres auteurs, dans la mesure où elle inclut fréquemment la stratégie de légitimation ou la stratégie collaborative, qui sont considérées ici de manière distincte.

Le brevet peut tout d'abord avoir une influence positive sur le niveau des ventes d'une entreprise, dans la mesure où il informe le consommateur de la qualité technologique d'un produit (Mitkova, 2005). Ainsi, les organisations telles que les firmes pharmaceutiques ou cosmétiques indiquent régulièrement l'existence de brevets sur l'emballage de leurs produits. Dans le même ordre d'idée, le brevet peut également avoir un impact positif sur la valorisation boursière des entreprises dans la mesure où il informe les investisseurs de la performance technologique d'une organisation (Ceccagnoli, 2009). Des travaux ont ainsi montré une corrélation positive entre la valeur d'une entreprise et la valeur du portefeuille de brevets qu'elle détient (Bloom \& Van Reenen, 2002; Hall, Jaffe, \& Trajtenberg, 2005).

\section{La stratégie de légitimation}


La stratégie de légitimation correspond à l'usage du brevet en tant que vecteur de réputation afin d'éviter un désavantage compétitif. La théorie néo-institutionnelle a depuis longtemps montré que les organisations sont davantage susceptibles de survivre si elles acquièrent une légitimité à l'égard des constituants de leur environnement (Baum \& Oliver, 1991). En particulier, les organisations jeunes ou de petite taille sont connues pour avoir particulièrement besoin d'une telle légitimité, car elle peuvent respectivement souffrir du " handicap de la jeunesse » (liability of newness) ou du " handicap de la petitesse » (liability of smallness) (Abatecola, Cafferata, \& Poggesi, 2012). Dans la mesure où le brevet est un titre délivré par des offices indépendants qui est censé garantir la nouveauté et la propriété intellectuelle de l'invention, il permet d'asseoir la légitimé des organisations en informant de la profitabilité potentielle de leurs activités de R\&D (Niosi, 2003). Alors que la stratégie de signal permet aux organisations d'être économiquement performantes, dans une stratégie de légitimation, le brevet facilite leur survie - la distinction entre les deux est donc une question de degré.

Dans la lignée de cette perspective néo-institutionnaliste, de nombreux travaux ont ainsi montré que le brevet peut significativement accroître l'accès des petites entreprises à des sources de financement, en particulier dans les industries des biotechnologies, qui sont particulièrement compétitives (Hsu \& Ziedonis, 2013; Levitas \& McFadyen, 2009; Niosi, 2003). Le brevet est aussi très utile lors des premières phases de financement des entreprises naissantes, lorsqu'il n'existe pas de mécanismes alternatifs permettant de donner des gages de crédibilité aux investisseurs externes (Conti, Thursby, \& Thursby, 2013; Freitas \& Nuvolari, 2012; Hsu \& Ziedonis, 2013; C. Long, 2002; Useche, 2015). Par la suite, une fois que l'asymétrie d'information entre les investisseurs et les entreprises financées diminuent, l'intérêt d'opérer un recours au brevet dans une stratégie de légitimation diminue (Hoenen, Kolympiris, Schoenmakers, \& Kalaitzandonakes, 2014).

\section{La stratégie collaborative}

La stratégie collaborative consiste à employer le brevet comme un vecteur de réputation afin de développer les échanges inter-organisationnels en matière d'innovation. Ici, le brevet est employé afin d'indiquer les compétences d'une organisation à ses partenaires potentiels et d'initier de la sorte des collaborations à n'importe quel moment du processus d'innovation. Dans un contexte d'innovation ouverte, ces collaborations constituent un enjeu de taille pour nombre d'organisations innovantes (Chesbrough, 2003).

Ainsi, le brevet peut aider les organisations à trouver un partenaire afin d'assurer la phase de recherche et de développement des innovations (Blind et al., 2006; Ernst, 2003; Freitas \& Nuvolari, 2012). De la même manière, le brevet permet également de trouver les fournisseurs d'une technologie particulière (Arora \& Gambardella, 2010). Dans cette perspective, les bases de données de brevets sont particulièrement utiles dans la mesure où elles permettent d'entreprendre des investigations systématiques (Blind et al., 2006; Ernst, 2003).

Il est généralement admis que le concept de réputation inclut à la fois le contenu de la représentation du comportement d'un acteur, l'intensité de la réception de cette représentation, ainsi que la valeur que l'on lui attribue (Lange, Lee, \& Dai, 2011). Il est intéressant de noter que l'usage du brevet en tant que vecteur de réputation a des limitations associées à chacune de ces trois dimensions. Premièrement, la qualité du contenu des brevets est parfois questionnée, d'une part en raison de stratégies rédactionnelles qui visent à augmenter le nombre de revendications qu'ils contiennent tout en limitant au maximum la 
diffusion d'informations sensibles, et d'autre part en raison de la hausse du nombre de brevets déposés (de la Potterie, 2011; Ouellette, 2012; van Zeebroeck, de la Potterie, \& Guellec, 2009). Deuxièmement, ces problèmes de quantité et de qualité des brevets suscitent en conséquence des préoccupations quant à leur capacité individuelle à diffuser efficacement des informations (Ouellette, 2012). Troisièmement, ces problèmes sont également susceptibles de compliquer la valorisation des brevets par les acteurs concernés (Angue, Ayerbe, \& Mitkova, 2013; Van Zeebroeck, 2011). Pour toutes ces raisons, le brevet ne constitue pas toujours un vecteur de réputation efficace ${ }^{8}$.

\section{Le brevet comme mécanisme de protection}

Dernier usage, mais non des moindres, le rôle de protection du brevet est probablement le plus connu, dans la mesure où il est à l'origine de sa création. Le dépôt de brevet confère en effet des droits de propriété intellectuelle, permettant ainsi aux organisations de contrôler leurs activités d'innovation au niveau inter-organisationnel en autorisant ou en interdisant la mise en œuvre d'activités d'innovation par des concurrents. De manière similaire au rôle de vecteur de réputation du brevet, trois stratégies génériques peuvent être distinguées en matière d'usage du brevet comme mécanisme de protection (Somaya, 2012).

\section{La stratégie propriétaire}

La stratégie propriétaire correspond à l'usage du brevet en tant que mécanisme de protection afin d'obtenir un avantage compétitif sur un marché (Somaya, 2012). Cet usage spécifique du brevet correspond à sa vocation historique, qui était de promouvoir l'innovation en fournissant aux inventeurs un intervalle de temps au cours duquel ils pourraient obtenir un retour sur leurs investissements (P. O. Long, 1991).

Si un unique brevet peut parfois être suffisant afin d'obtenir un pouvoir de marché, il faut bien souvent déposer toute une série de brevets pour que l'avantage compétitif obtenu soit réel. Le recours à de multiple brevets peut prendre différentes formes. Par exemple, une barrière de brevets (patent fence) peut être établie afin de bloquer un champ de R\&D - ceci est également appelé parfois "stratégie préemptive" (Ceccagnoli, 2009) -, une inondation de brevets (patent flooding) peut être employée afin de rendre une zone de R\&D difficile d'accès, et un encerclement de brevets (patent surrounding) peut être mis en œuvre afin de contrôler des zones de R\&D complémentaires à une technologie particulière (Granstrand, 1999). Alors que les barrières de brevets sont souvent employées afin d'exclure les compétiteurs dans le cas de technologies dites discrètes, l'établissement de maquis de brevets au travers d'inondations ou d'encerclements de brevets est davantage susceptible de se produire dans le cas de technologies complexes, dans la mesure où les zones de R\&D sont plus difficiles à bloquer (Shapiro, 2001; Von Graevenitz, Wagner, \& Harhoff, 2011) .

\section{La stratégie défensive}

\footnotetext{
8 Pour une analyse détaillée des causes et des conséquences de ce problème, voir Pénin et Neicu (2018).

9 Les technologies discrètes sont des technologies comprenant un nombre limité de sous-systèmes, et dont la protection nécessite donc un nombre de brevets limité - c'est le cas dans l'industrie pharmaceutique -, par opposition aux technologies complexes - telles que celles de l'industrie électronique - qui comprennent un grand nombre de sous-systèmes et dont la protection nécessite de nombreux brevets (Hall \& Ziedonis, 2001; Levin et al., 1987).
} 
La stratégie défensive correspond à l'usage du brevet en tant que mécanisme de protection afin d'éviter un désavantage compétitif (Somaya, 2012). Cette stratégie vise à préserver la liberté d'une organisation à utiliser des brevets; elle est généralement employée lorsqu'il existe une incertitude quant à la profitabilité future de zones technologiques (Somaya, 2012) ${ }^{10}$. La stratégie défensive peut être mise en œuvre de deux manières (Somaya, 2012). Le brevet peut tout d'abord être directement utilisé afin de prévenir un désavantage compétitif. Ici, les mêmes pratiques que celles employées dans une stratégie propriétaire peuvent être utilisées : si un unique brevet est parfois suffisant, plusieurs brevets sont souvent nécessaires afin de protéger les inventeurs d'un désavantage compétitif. Ainsi, des barrières de brevets, des inondations de brevets, ou des encerclements de brevets peuvent également être employés dans le cadre de cette stratégie (Granstrand, 1999). Si ces brevets s'avèrent profitables, ils pourront ensuite être utilisés selon une stratégie propriétaire précédemment présentée.

Le brevet peut également servir à prévenir un désavantage compétitif de manière indirecte. Lorsqu'il n'est pas possible d'identifier ex ante les zones technologiques qui seront profitables, le dépôt massif de brevets permet par la suite de réaliser des cessions de licences croisées ou de mettre en place des situations de hold-up mutuels ${ }^{11}$ auprès des organisations qui détiennent les technologies convoitées, et de s'assurer ainsi de la possibilité d'exploiter ultérieurement ces technologies (Somaya, 2012). Les brevets sont ainsi employés dans le cadre d'une stratégie de négociation que nous développons ci-après.

\section{La stratégie de négociation}

La stratégie de négociation correspond à l'usage du brevet en tant que mécanisme de protection afin de générer des rentes au travers d'échanges inter-organisationnels (Somaya, 2012). Alors que la stratégie propriétaire vise à bénéficier directement des droits qui sont conférés par la propriété intellectuel au travers de l'exploitation de l'invention, la stratégie de négociation consiste à utiliser la propriété intellectuelle comme monnaie d'échange. De la même manière que pour la stratégie défensive, la stratégie de négociation peut être mise en œuvre de manière directe ou indirecte (Somaya, 2012).

Premièrement, le brevet peut directement procurer des rentes à une organisation au travers de la cession de brevets ou de la cession de licences, en particulier lorsqu'il s'agit de technologies qui ne sont pas centrales dans les activités de l'organisation en question (Somaya, 2012). Avec l'accroissement de la division de la production et de l'utilisation des technologies entre les organisations, les échanges de technologies ont explosé depuis les années 1980, et représentent actuellement des milliards de dollars (Arora \& Gambardella, 2010). De telles pratiques sont communes dans le secteur des technologies de l'information et de la communication ou dans celui de l'industrie pharmaceutique, qui sont particulièrement innovants ${ }^{12}$.

10 Le droit français limite le recours à cette pratique : le propriétaire d'un brevet a l'obligation d'exploiter son brevet dans un délai de trois ans après sa délivrance, sous peine d’être obligé d’octroyer une licence à quiconque en ferait la demande.

11 Une situation de hold-up mutuel survient lorsque deux organisations s’accordent réciproquement le droit d'exploiter leurs technologies à défaut de se poursuivre en infraction.

12 Il est intéressant de noter que, depuis les années 1990, la stratégie de négociation a donné lieu à l'apparition d’organisations spécialisées dans l'acquisition et la défense de la propriété intellectuelle. Appelées trolls des brevets (patent trolls) ou requins des brevets (patent sharks), ces entités ont été critiquées en raison des sommes importantes qu'elles réclament parfois aux organisations coupables de violation de propriété intellectuelle. Néanmoins, elles ont également été reconnues avoir un rôle positif dans la mesure où elles permettent la défense des droits de propriété intellectuelle et le recouvrement des montants dus à des organisations qui ne sont pas en 
Par-delà les cessions individuelles, les cessions de licences peuvent également être réalisées au travers de la création d'un consortium chargé de regrouper en packages des ensembles de brevets détenues par de multiples organisations. Chacun des membres du consortium peut alors utiliser les brevets détenus par ses partenaires dans une logique de licence croisée précédemment évoquée. L'apparition de ces pools de brevets fait souvent suite à l'établissement de standards technologiques qui requièrent le recours à des brevets complémentaires de manière conjointe. C'est par exemple le cas de l'établissement du standard DVD ROM (Layne-Farrar \& Lerner, 2011).

Deuxièmement, le brevet peut indirectement procurer des rentes à une organisation au travers de diverses négociations. Somaya (2012) rapporte ainsi le cas d'une organisation détentrice de brevets dont les droits de propriété intellectuelle avaient été enfreints par un client qui souhaitait changer de fournisseur. Cette organisation avait renouvelé sont contrat d'approvisionnement avec ledit client en le menaçant de poursuites en justice relatives à l'infraction si le contrat ne lui était pas ré-attribué. Dans le même ordre d'idées, et de manière plus générale, on peut noter que les brevets sont dorénavant employés dans diverses négociations financières. Par exemple, les brevets sont de plus en plus employés en tant que garantie dans l'obtention d'un prêt (Amable, Chatelain, \& Ralf, 2010). De manière similaire, les brevets sont de plus en plus utilisés afin de réduire les impôts sur les sociétés, de nombreuses mesures fiscales incitatives en matière de R\&D appelées boites à brevets (patent boxes) ayant été prises dans différents pays afin de réduire le taux d'imposition sur les revenus issus des brevets (Atkinson \& Andes, 2011).

Si le brevet peut constituer un instrument de protection utile, cet usage souffre d'importantes limites. Premièrement, afin d'être brevetable, les inventions doivent être nouvelles, issues d'une activité inventive, et susceptibles d'applications industrielles, ce qui n'est pas toujours le cas. Deuxièmement, le nombre de dépôts de brevets et la taille croissante de ces derniers conduisent parfois à l'attribution de brevets de faible qualité, ce qui induit un risque accru d'invalidation. Troisièmement, l'obtention et la défense des brevets sont coûteux, et les organisations - en particulier les Petites et Moyennes Entreprises - ne sont pas toujours en mesure de prendre en charge ces coûts (Friedman, Landes, \& Posner, 1991). Quatrièmement, les retours sur investissement ne couvrent pas toujours les coûts du brevet, lequel devient alors non rentable (Friedman et al., 1991). Cinquièmement, la révélation des inventions au travers des brevets peut se révéler contre-productive lorsque la durée de protection offerte par le brevet est inférieure à la durée nécessaire pour répliquer une invention, ou lorsque le brevet signale des zones de recherche technologique profitables au concurrents (Friedman et al., 1991). Pour toutes ces raisons, les inventeurs peuvent ne pas être en mesure de recourir au brevet pour protéger leurs innovations et doivent alors employer des mécanismes de protection alternatifs (pour une recension des mécanismes de protection des innovations contre l'imitation, voir Mandard, 2016).

\section{DISCUSSION}

Dans cet article, nous avons proposé un cadre analytique qui répertorie les usages du brevet. Pour ce faire, nous avons remis en question la définition traditionnelle du brevet comme un mécanisme de protection des innovations pour le définir plus largement comme un

mesure de s'engager dans des contentieux, ce qui est notamment le cas des petites entreprises (pour un panorama de ce sujet, voir Geradin, Layne-Farrar, \& Padilla, 2011; Pohlmann \& Opitz, 2013). 
mécanisme de gouvernance des activités d'innovation des organisations, au niveau intracomme inter-organisationnel (Tableau 1). Nous avons de la sorte montré que le brevet a quatre principaux usages: indicateur d'activité, outil de motivation, vecteur de réputation ou mécanisme de protection. En suivant ce raisonnement, il est intéressant de noter que, de nos jours, désigner le brevet comme un mécanisme de protection des innovations, c'est opérer une synecdoque, puisque cela revient à désigner le tout par l'une de ses parties. Nous avons ensuite détaillé successivement les modalités de mise en œuvre de ces différents usages ainsi que leurs limites.

Le cadre d'analyse développé dans cet article est résumé dans l'arbre de décision en Figure 1. Pour autant que nous le sachions, cet arbre de décision recense et articule l'ensemble des usages connus du brevet et leurs principales modalités de mise en œuvre, ces différents usages pouvant bien entendu être déployés de manière complémentaire. 
Figure 1-Les usages du brevet : un arbre de décision ouvre

Benchmarking interne

Indicateur de performance 
Stratégie de négociation

\section{Implications scientifiques}

D'un point de vue scientifique, le cadre analytique proposé pourrait permettre de structurer la recherche sur les brevets.

Il pourrait tout d'abord être employé afin de répertorier les connaissances accumulées. On peut ainsi noter d'emblée que le rôle de mécanisme de protection des brevets a été particulièrement étudié, tandis que des usages tels que celui de vecteur de réputation ou ses usages intra-organisationnels ont été peu explorés, étant même parfois jugés comme secondaires (Somaya, 2012).

En conséquence, ce cadre analytique pourrait susciter la conduite de recherches complémentaires. Premièrement, les antécédents et les conséquences des usages du brevet pourraient être davantage étudiées. Tout d'abord, de nouveaux travaux sur les usages du brevet en tant qu'indicateur de performance, outil de motivation ou vecteur de réputation pourraient être réalisés, dans la mesure où ces usages ont fait l'objet de moins d'attention. Également, en matière d'usage du brevet, des variations dans le type d'innovation considéré pourraient être examinées. Des travaux antérieurs ont en effet montré que les motifs qui conduisent à breveter des innovations produits sont susceptibles de différer de ceux qui conduisent à des innovations procédés (Cohen, Nelson, \& Walsh, 2000). Des variations dans le type d'acteur considéré pourraient aussi être examinées. Par exemple, des travaux ont montré que de grandes entreprises telles que Air Liquide ou Thalès ont mis en place des services spécifiquement dédiés au management de leurs portefeuilles de brevets (Ayerbe, Lazaric, Callois, \& Mitkova, 2014; Ayerbe \& Mitkova, 2005). De nouvelles recherches pourraient viser à identifier les entreprises qui mettent en œuvre ces structures et l'influence de ces dernières sur les usages des brevets. Enfin, la place du brevet parmi les autres instruments de gouvernance des activités d'innovation des organisations, tels que des règles (formelles ou informelles), ou d'autres outils de mesure des activités d'innovation (dépenses de R\&D, publications scientifiques, etc.) serait une piste de recherche intéressante.

Deuxièmement, des relations de substitution ou de complémentarité entre les différents usages du brevet pourraient être plus précisément identifiées. À titre d'exemple, une recherche récente a montré que les brevets pouvaient être simultanément employés en suivant une stratégie propriétaire et une stratégie de légitimation (Hsu \& Ziedonis, 2013). De manière similaire, il a été montré que les brevets peuvent venir simultanément soutenir la motivation intrinsèque et la motivation extrinsèque des inventeurs (Chevreuil et al., 2012). Ceci est un courant de recherche émergent qui mériterait d'être développé. Il pourrait enfin être 
intéressant d'identifier des hiérarchisations dans les usages du brevet : quels sont les usages principaux et les usages accessoires ? Si la protection des retombées économiques des innovations est souvent la principale cause du dépôt de brevets, il semblerait que cet usage soit parfois secondaire, la priorité pouvant être donnée à la diffusion de la réputation de l'entreprise en matière d'innovation ou au développement d'une culture de l'innovation en interne.

\section{Implications managériales}

D'un point de vue pratique, deux grandes tendances se dessinent en matière d'usage des brevets. D'une part, il est connu que les usages internes sont généralement moins fréquents que ceux inter-organisationnels (Blind et al., 2006; Cohen et al., 2000). D'autre part, ces usages internes sont également davantage mis en œuvre par les grandes organisations que par les petites et moyennes entreprises (Blind et al., 2006; Graham \& Sichelman, 2008), en raison notamment d'une méconnaissance des usages internes des brevets de la part de ces dernières (Petit, Dubois, Harand, \& Quazzotti, 2011; Pitkethly, 2012). Ces constats ont récemment conduit au développement de différentes initiatives visant à promouvoir la question de la propriété intellectuelle, avec le lancement du projet IPeuropAware en Europe ou la mise au point du IP Awareness Assessment Tool aux États-Unis. Dans la lignée de ces préoccupations, il nous semble qu'un cadre d'analyse tel que celui que nous proposons ici pourrait être intéressant pour les détenteurs de brevets, dans la mesure où il leur offre un vade-mecum accessible. Dans l'idéal, ce cadre d'analyse pourrait être fourni par les offices de brevets aux inventeurs afin de les informer des nombreuses fonctionnalités que leurs brevets sont susceptibles de leur procurer. 


\section{RÉFÉRENCES}

ABATECOLA, G., CAFFERATA, R., POGGESI, S. (2012), Arthur Stinchcombe's "liability of newness": contribution and impact of the construct, Journal of Management History, 18(4), 402-418.

AGARWAL, R., GANCO, M., ZIEDONIS, R. H. (2009), Reputations for toughness in patent enforcement: Implications for knowledge spillovers via inventor mobility, Strategic Management Journal, 30(13), 1349-1374.

ALMEIDA, P., KOGUT, B. (1999), Localization of knowledge and the mobility of engineers in regional networks, Management Science, 45(7), 905-917.

AMABILE, T. M. (1983), The social psychology of creativity: A componential conceptualization, Journal of Personality and Social Psychology, 45(2), 357.

AMABLE, B., CHATELAIN, J.-B., RALF, K. (2010), Patents as collateral, Journal of Economic Dynamics and Control, 34(6), 1092-1104.

ANGUE, K., AYERBE, C., MITKOVA, L. (2013), A method using two dimensions of the patent classification for measuring the technological proximity: an application in identifying a potential R\&D partner in biotechnology, The Journal of Technology Transfer, 39(5), 716-747. ARORA, A., GAMBARDELLA, A. (2010), Ideas for rent: An overview of markets for technology, Industrial and Corporate Change, 19(3), 775-803.

ATKINSON, R. D., ANDES, S. M. (2011), Patent boxes: innovation in tax policy and tax policy for innovation, ITIF, October, http://www.itif.org/files/2011-pb-atkinson.pdf

AYERBE, C., LAZARIC, N., CALLOIS, M., MITKOVA, L. (2014), The new challenges of organizing intellectual property in complex industries: A discussion based on the case of Thales, Technovation, 34(4), 232-241.

AYERBE, C., MITKOVA, L. (2005), Quelle organisation pour la valorisation des brevets d'invention? Revue Française de Gestion, 155, 191-206.

BALDINI, N., GRIMALDI, R., SOBRERO, M. (2007), To patent or not to patent? A survey of Italian inventors on motivations, incentives, and obstacles to university patenting, Scientometrics, 70(2), 333-354.

BAUM, J. A., OLIVER, C. (1991), Institutional linkages and organizational mortality, Administrative Science Quarterly, 36(2), 187-218.

BLIND, K., EDLER, J., FRIETSCH, R., SCHMOCH, U. (2006), Motives to patent: Empirical evidence from Germany, Research Policy, 35(5), 655-672.

BLOOM, N., VAN REENEN, J. (2002), Patents, Real Options and Firm Performance, The Economic Journal, 112(478), C97-C116.

CECCAGNOLI, M. (2009), Appropriability, preemption, and firm performance, Strategic Management Journal, 30(1), 81-98.

CHESBROUGH, H. W. (2003), Open innovation: The new imperative for creating and profiting from technology, Harvard Business Press.

CHEVREUIL, S., CORBEL, P., MBONGUI-KIALO, S. (2012), Le brevet comme input dans le processus d'innovation: le cas Peugeot-Citroën Automobiles, In Les nouvelles fonctions du brevet (pp. 45-61). Paris: Economica.

COHEN, W. M., NELSON, R. R., WALSH, J. P. (2000), Protecting their intellectual assets: Appropriability conditions and why U.S. manufacturing firms patent (or not) (SSRN Scholarly Paper No. ID 214952), Rochester, NY: Social Science Research Network.

COHENDET, P., FARCOT, M., PÉNIN, J. (2006), Entre incitation et coordination : repenser le rôle économique du brevet d'invention dans une économie fondée sur la connaissance, Management International, 10, 65-84. 
CONTI, A., THURSBY, J., THURSBY, M. (2013), Patents as signals for startup financing, The Journal of Industrial Economics, 61(3), 592-622.

CORBEL, P., FERNANDEZ, F., GENDRAUD, P. (2007), Le budget comme relais de la stratégie: le cas du brevet, In Actes de la XVI ème Conférence Internationale de l'AIMS.

CORBEL, P., LE BAS, C. (2011), Les nouvelles fonctions du brevet: approches économiques et managériales, Economica.

DE LA POTTERIE, B. VAN P. (2011), The quality factor in patent systems, Industrial and Corporate Change, 20(6), 1755-1793.

DE MAN, A.-P., DUYSTERS, G. (2005), Collaboration and innovation: A review of the effects of mergers, acquisitions and alliances on innovation, Technovation, 25(12), 13771387.

DELERUE, H., LEJEUNE, A. (2010), Job mobility restriction mechanisms and appropriability in organizations: The mediating role of secrecy and lead time, Technovation, 30(5-6), 359-366.

EDSTRÖM, A., GALBRAITH, J. R. (1977), Transfer of managers as a coordination and control strategy in multinational organizations, Administrative Science Quarterly, 22(2), 248263.

ERNST, H. (1998), Patent portfolios for strategic R\&D planning, Journal of Engineering and Technology Management, 15(4), 279-308.

ERNST, H. (2003), Patent information for strategic technology management, World Patent Information, 25(3), 233-242.

ERNST, H., LEPTIEN, C., VITT, J. (2000), Inventors are not alike: The distribution of patenting output among industrial R\&D personnel, Engineering Management, IEEE Transactions On, 47(2), 184-199.

ERNST, H., OMLAND, N. (2011), The Patent Asset Index-A new approach to benchmark patent portfolios, World Patent Information, 33(1), 34-41.

FABRY, B., ERNST, H., LANGHOLZ, J., KÖSTER, M. (2006), Patent portfolio analysis as a useful tool for identifying R\&D and business opportunities - an empirical application in the nutrition and health industry, World Patent Information, 28(3), 215-225.

FREITAS, I. M. B., NUVOLARI, A. (2012), Traditional Versus Heterodox Motives for Academic Patenting: Evidence from the Netherlands, Industry and Innovation, 19(8), 671695.

FRIEDMAN, D. D., LANDES, W. M., POSNER, R. A. (1991), Some economics of trade secret law, The Journal of Economic Perspectives, 5(1), 61-72.

GANCO, M., ZIEDONIS, R. H., AGARWAL, R. (2015), More stars stay, but the brightest ones still leave: Job hopping in the shadow of patent enforcement, Strategic Management Journal, 36(5), 659-685.

GERADIN, D., LAYNE-FARRAR, A., PADILLA, A. J. (2011), Elves or Trolls? The role of nonpracticing patent owners in the innovation economy, Industrial and Corporate Change, 21(1), 73-94.

GÖKTEPE-HULTEN, D., MAHAGAONKAR, P. (2010), Inventing and patenting activities of scientists: In the expectation of money or reputation? The Journal of Technology Transfer, 35(4), 401-423.

GRAHAM, S. J., SICHELMAN, T. (2008), Why do start-ups patent? Berkeley Technology Law Journal, 23, 1063.

GRANSTRAND, O. (1999), The economics and management of intellectual property towards intellectual capitalism, Edward Elgar Publishing. 
GREENBERG, J. (1990), Organizational justice: Yesterday, today, and tomorrow, Journal of Management, 16(2), 399-432.

GUPTA, P. P., DIRSMITH, M. W., FOGARTY, T. J. (1994), Coordination and control in a government agency: Contingency and institutional theory perspectives on GAO audits, Administrative Science Quarterly, 39(2), 264-284.

HAEUSSLER, C., SAUERMANN, H. (2013), Credit where credit is due? The impact of project contributions and social factors on authorship and inventorship, Research Policy, 42(3), 688-703.

HALL, B. H., JAFFE, A., TRAJTENBERG, M. (2005), Market value and patent citations, RAND Journal of Economics, 36(1), 16-38.

HALL, B. H., ZIEDONIS, R. H. (2001), The patent paradox revisited: an empirical study of patenting in the US semiconductor industry, 1979-1995, RAND Journal of Economics, 32(1), 101-128.

HARHOFF, D., HOISL, K. (2007), Institutionalized incentives for ingenuity-Patent value and the German Employees' Inventions Act, Research Policy, 36(8), 1143-1162.

HOENEN, S., KOLYMPIRIS, C., SCHOENMAKERS, W., KALAITZANDONAKES, N. (2014), The diminishing signaling value of patents between early rounds of venture capital financing, Research Policy, 43(6), 956-989.

HOLGERSSON, M. (2013), Patent management in entrepreneurial SMEs: A literature review and an empirical study of innovation appropriation, patent propensity, and motives, $R \& D$ Management, 43(1), 21-36.

HSU, D. H., ZIEDONIS, R. H. (2013), Resources as dual sources of advantage: Implications for valuing entrepreneurial-firm patents, Strategic Management Journal, 34(7), 761-781.

KUMAR, S., SETH, A. (1998), The design of coordination and control mechanisms for managing joint venture-parent relationships, Strategic Management Journal, 19(6), 579-599.

LANGE, D., LEE, P. M., DAI, Y. (2011), Organizational reputation: A review, Journal of Management, 37(1), 153-184.

LANGINIER, C. (2005), Using patents to mislead rivals, Canadian Journal of Economics, $38(2), 520-545$.

LAYNE-FARRAR, A., LERNER, J. (2011), To join or not to join: Examining patent pool participation and rent sharing rules, International Journal of Industrial Organization, 29(2), 294-303.

LEE, S., YOON, B., LEE, C., PARK, J. (2009), Business planning based on technological capabilities: Patent analysis for technology-driven roadmapping, Technological Forecasting and Social Change, 76(6), 769-786.

LEE, S., YOON, B., PARK, Y. (2009), An approach to discovering new technology opportunities: Keyword-based patent map approach, Technovation, 29(6-7), 481-497.

LEVIN, R. C., KLEVORICK, A. K., NELSON, R. R., WINTER, S. G., GILBERT, R., GRILICHES, Z. (1987), Appropriating the returns from industrial research and development, Brookings Papers on Economic Activity, 1987(3), 783-831.

LEVITAS, E., MCFADYEN, M. (2009), Managing liquidity in research-intensive firms: Signaling and cash flow effects of patents and alliance activities, Strategic Management Journal, 30(6), 659-678.

LONG, C. (2002), Patent signals, The University of Chicago Law Review, 625-679.

LONG, P. O. (1991), Invention, authorship," intellectual property," and the origin of patents: Notes toward a conceptual history, Technology and Culture, 32(4), 846-884.

MALHOTRA, D., LUMINEAU, F. (2011), Trust and collaboration in the aftermath of conflict: The effects of contract structure, Academy of Management Journal, 54(5), 981-998. 
MANDARD, M. (2016), Les mécanismes de protection des innovations contre l'imitation: un cadre d'analyse générique et un inventaire, Gérer et Comprendre, 3, 32-40.

MARSDEN, P. V., COOK, C. R., KALLEBERG, A. L. (1994), Organizational structures coordination and control, American Behavioral Scientist, 37(7), 911-929.

MITKOVA, L. (2005), Marketing: A key element in patent management, International Journal of Technology Transfer and Commercialisation, 4(4), 487-499.

MOEHRLE, M. G., WALTER, L., GERITZ, A., MÜLLER, S. (2005), Patent-based inventor profiles as a basis for human resource decisions in research and development, $R \& D$ Management, 35(5), 513-524.

NELSON, A. J. (2009), Measuring knowledge spillovers: What patents, licenses and publications reveal about innovation diffusion, Research Policy, 38(6), 994-1005.

NIOSI, J. (2003), Alliances are not enough explaining rapid growth in biotechnology firms, Research Policy, 32(5), 737-750.

OUELLETTE, L. L. (2012), Do Patents Disclose Useful Information? Harvard Journal of Law and Technology, 25, 531.

PÉNIN, J. (2005), Patents versus ex post rewards: A new look, Research Policy, 34(5), 641656.

PÉNIN, J., NEICU, D. (2018), Patents and open innovation: Bad fences do not make good neighbors, Journal of Innovation Economics \& Management, 25, 57-85.

PETIT, C., DUBOIS, C., HARAND, A., QUAZZOTTI, S. (2011), A new, innovative and marketable IP diagnosis to evaluate, qualify and find insights for the development of SMEs IP practices and use, based on the AIDA approach, World Patent Information, 33(1), 42-50.

PITKETHLY, R. H. (2012), Intellectual property awareness, International Journal of Technology Management, 59(3/4), 163-179.

POHLMANN, T., OPITZ, M. (2013), Typology of the patent troll business, $R \& D$ Management, 43(2), 103-120.

RYAN, R. M., DECI, E. L. (2000), Intrinsic and extrinsic motivations: Classic definitions and new directions, Contemporary Educational Psychology, 25(1), 54-67.

SHAPIRO, C. (2001), Navigating the patent thicket: Cross licenses, patent pools, and standard setting, In Innovation Policy and the Economy (Vol. 1, pp. 119-150), MIT press.

SOMAYA, D. (2012), Patent strategy and management: An integrative review and research agenda, Journal of Management, 38(4), 1084-1114.

USECHE, D. (2015), Patenting Behaviour and the Survival of Newly Listed European Software Firms, Industry and Innovation, 22(1), 37-58.

VAN ZEEBROECK, N. (2011), The puzzle of patent value indicators, Economics of Innovation and New Technology, 20(1), 33-62.

VAN ZEEBROECK, N., DE LA POTTERIE, B. VAN P., GUELLEC, D. (2009), Claiming more: the increased voluminosity of patent applications and its determinants, Research Policy, 38(6), 1006-1020.

VON GRAEVENITZ, G., WAGNER, S., HARHOFF, D. (2011), How to measure patent thickets - A novel approach, Economics Letters, 111(1), 6-9. 